\title{
Erupción neonatal vesículo-pustular relacionada con trastorno mieloproliferativo transitorio en dos recién nacidos con trisomía 21
}

\author{
Vesiculopustular eruption associated with transient \\ myeloproliferative disorder in two newborns with trisomy 21 \\ Marina S. Meneses*, Florencia Galdeano, Emilce Baiardi, Sabrina Garcia-Amore, Gabriela Aprea, \\ Silvia Moreno y Marisel Garay \\ Servicio de Dermatología, Hospital Pediátrico Humberto Notti, Mendoza, Argentina
}

\section{Resumen}

Los niños con síndrome de Down (SD) tienen mayor riesgo de desarrollar el trastorno mieloproliferativo transitorio (TMT), que se caracteriza por pancitopenia, hepatoesplenomegalia y blastos en sangre periférica, con regresión en los primeros meses de vida. La afectación cutánea ronda el 5\%. Se presentan dos recién nacidos (RN) con trisomía 21, que a pocos días de vida desarrollaron lesiones cutáneas, de tipo pápulo-vesículas y pústulas, en rostro y tronco, vinculadas con hiperleucocitosis con blastos en sangre periférica. A pesar del curso benigno y autorresolutivo del TMT, estos pacientes deben controlarse de por vida, ya que tienen mayor riesgo de desarrollar leucemia.

Palabras clave: Erupción vesículo-pustular. Síndrome de Down. Trastorno mieloproliferativo transitorio.

\begin{abstract}
Children with Down syndrome (DS) are at increased risk of developing transient myeloproliferative disorder (TMD). This is characterized by pancytopenia, hepatosplenomegaly and blasts in peripheral blood, with regression in the first months of life. Skin involvement is around 5\%. We present two newborns (NBs) with Trisomy 21, a few days old who develop skin lesions, papulo-vesicle type and pustules, on the face and trunk, associated with hyperleukocytosis with blasts in peripheral blood. Despite the benign and self-solving course of TMD, these patients should be monitored for life, as they are at increased risk of developing leukemia.
\end{abstract}

Key words: Vesiculopustular eruption. Down syndrome. Transient myeloproliferative disorder.

Disponible en internet: 06-10-2021 Med Cutan Iber Lat Am. 2021;49(Suppl 1):61-66 www.MedicinaCutanealLA.com licencia CC BY-NC-ND (http://creativecommons.org/licenses/by-nc-nd/4.0/). 


\section{Introducción}

EI TMT es una enfermedad poco frecuente que describieron por primera vez Schunk y Lehman en $1954^{1}$. Por lo regular afecta a casi $10 \%$ de los recién nacidos con trisomía del par 21, los niños afectados pueden ser asintomáticos y sólo tienen blastos en sangre periférica, mientras que los casos más graves pueden presentarse con signos como hepatoesplenomegalia y fibrosis hepática ${ }^{2,3}$.

Si bien se ha señalado que puede acompañarse de manifestaciones cutáneas hasta en el $50 \%$ de los casos, existen muy pocas comunicaciones al respecto en las publicaciones médicas. Las lesiones cutáneas aparecen al nacimiento o en los primeros días de vida. Afectan en particular a la cara, sobre todo mejillas, seguido de tronco y extremidades, en forma de pápulas, vesículas, pústulas, o lesiones similares a las del impétigo. En dos tercios de los casos, la erupción disminuye en el primer mes $^{3}$.

A pesar de que se trata de una entidad benigna y de carácter autorresolutivo, existe la posibilidad de que evolucione a leucemia megacarioblástica aguda en los años siguientes, por lo que debe mantenerse un exhaustivo seguimiento de estos pacientes ${ }^{2,3}$.

Se presentan a continuación los casos de dos neonatos con SD que desarrollaron TMT con manifestaciones cutáneas.

\section{Caso clínico 1}

Paciente de género masculino, recién nacido de término, con peso adecuado para la edad gestacional $(2,750 \mathrm{~g})$. Por sospecha de trisomía del par 21 se solicitó cariotipo que confirmó dicha alteración genética.

Al día 19 de vida ingresa al servicio de neonatología con sospecha de sepsis tardía, con hemocultivos positivos para $S$. epidermidis, y se lo trata con meropenem-vancomicina por 13 días. Los hemogramas mostraron leucocitosis con blastos (30\%) y se detectó hepatomegalia.

Al noveno día de internación presentó una dermatosis que afectaba sobre todo al tronco y las mejillas, la cual consistía en lesiones pápulo-vesiculares tensas, de contenido claro, confluentes, que conformaban placas sobre una base eritematosa. Éstas se acompañaban de pústulas, algunas similares a las del impétigo y áreas exulceradas. No hubo respuesta frente al tratamiento con antibióticos tópicos (Figs. 1 y 2).

Los hemogramas mostraron porcentaje de blastos en aumento, hasta valores de $78 \%$ (Tabla 1), por lo

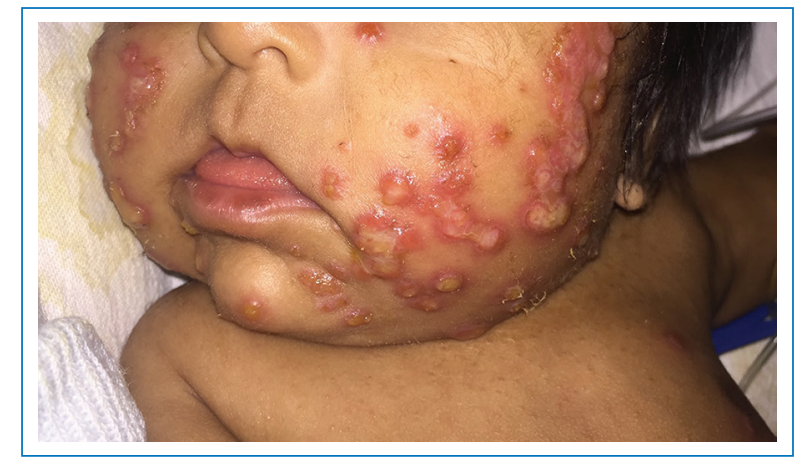

Figura 1. Caso 1. Múltiples vesículas y pústulas confluentes, algunas con costra amarillenta en la superficie similares a las del impétigo, que se asientan sobre una base eritematosa, localizadas en el rostro.

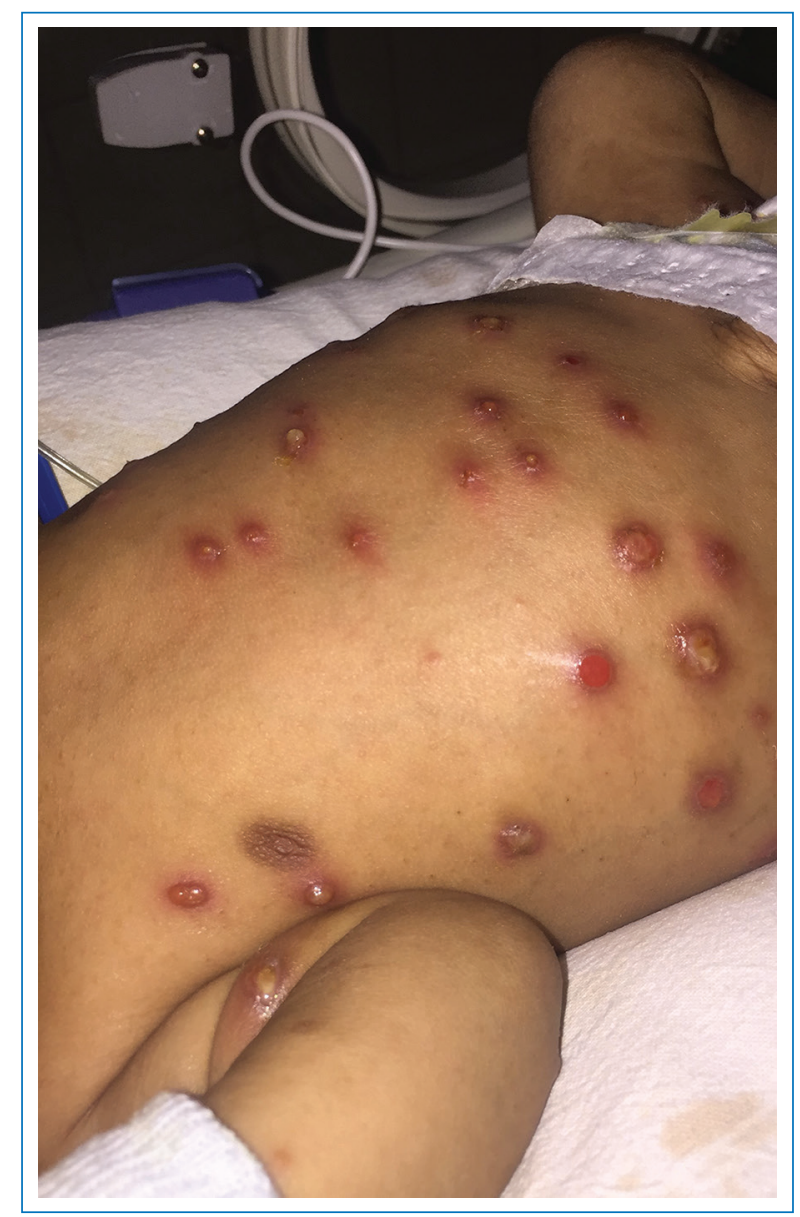

Figura 2. Caso 1. Vesículas, ampollas y pústulas, algunas destechadas, a nivel del tronco.

que se solicitó citometría de flujo en sangre periférica y biopsia cutánea para estudio histopatológico. La citometría mostró blastos mieloides $(52 \%$ de blastos de linaje mieloide; CD45 + débil, CD34 +, CD117 +, 
Tabla 1. Evolución en los hemogramas del caso clínico 1

\begin{tabular}{|l|c|c|c|c|c|c|}
\hline HxH & Día 1 & Día 2 & Día 3 & Día 10 & Día 14 & Día 35 \\
\hline Leucocitos totales & 40,000 & 35,000 & 36,000 & 22,000 & 16,500 & 12,600 \\
\hline Eosinófilos & & & & & & $3 \%$ \\
\hline Basófilos & & & & $2 \%$ & & \\
\hline Baciliformes & & & & $2 \%$ & & $3 \%$ \\
\hline Segmentados & $12 \%$ & $8 \%$ & $16 \%$ & $4 \%$ & $12 \%$ & $21 \%$ \\
\hline Linfocitos & $21 \%$ & $8 \%$ & $3 \%$ & $8 \%$ & $58 \%$ & $47 \%$ \\
\hline Monocitos & $3 \%$ & $6 \%$ & $7 \%$ & $0 \%$ & & $4 \%$ \\
\hline Blastos & $63 \%$ & $78 \%$ & $74 \%$ & $84 \%$ & $30 \%$ & $22 \%$ \\
\hline Mielocitos & $1 \%$ & & & & & \\
\hline
\end{tabular}

HxH: hemograma por hematólogo.

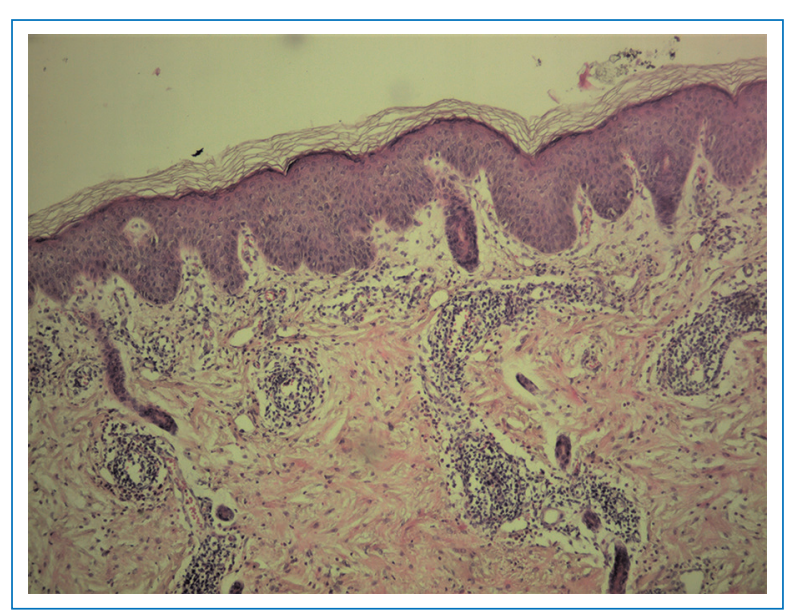

Figura 3. Caso 1. Anatomía patológica (H-E, 10x). Hiperqueratosis. Infiltrado dérmico perivascular moderado con extensión a TCS, de tipo mixto.

HLA DR +, 13 het 33 + parcial, 56 + parcial) con diagnóstico de TMT. En la biopsia de piel (Figs. 3 y 4) se identificó en la piel un moderado infiltrado perivascular que se extendía a hipodermis, constituido por polimorfonucleares, eosinófilos y elementos celulares que mostraban nidos irregulares e hipercromáticos (blastos).

Se adoptó una conducta expectante y el paciente fue seguido con hemogramas por hematólogo periódicos, con normalización de éstos hacia los tres meses de vida, en concordancia con la desaparición de las lesiones cutáneas. Hasta ahora, y luego de dos años después del inicio de la enfermedad, el paciente persiste con controles normales.

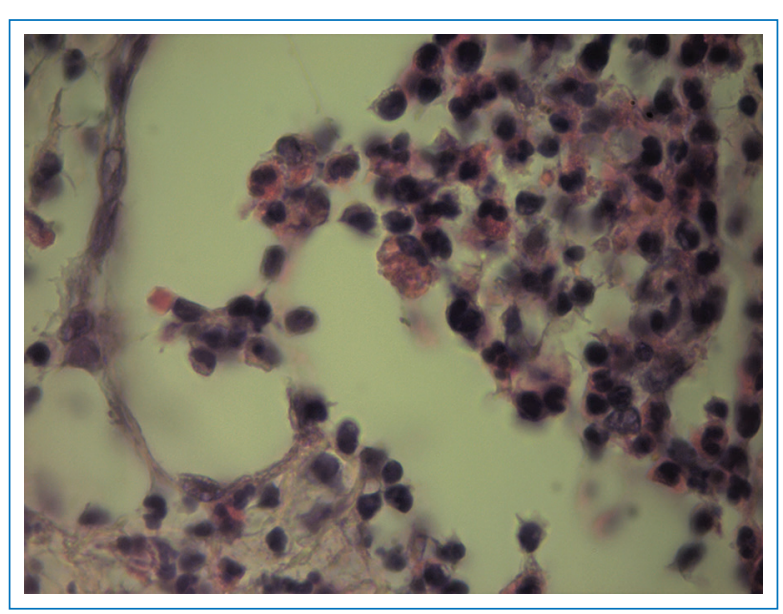

Figura 4. Caso 1. Anatomía patológica (H-E, 100x). Infiltrado constituido por leucocitos PMN, eosinófilos, histiocitos y blastos aislados.

\section{Caso clínico 2}

Recién nacido de término de sexo masculino, con peso adecuado para la edad gestacional (2,600 gr), con diagnóstico de síndrome de Down. Desarrolló durante los primeros días de vida hiperleucocitosis con $25 \%$ de blastos en sangre periférica (Tabla 2).

En el plano cutáneo mostró lesiones pequeñas de tipo pápulo-vesicular, algunas con costras melicéricas en superficie, áreas de exulceración, asentadas sobre una base eritematosa. Dicha dermatosis se diseminaba a mejillas, tronco y miembros superiores (Fig. 5).

Fue objeto de biopsia de piel y citometría de sangre periférica con similares hallazgos a los del caso 1 , de modo que se estableció el diagnóstico de TMT. 
Tabla 2. Evolución de los hemogramas del caso clínico 2

\begin{tabular}{|l|c|c|c|c|c|}
\hline HxH & Día 1 & Día 2 & Día 5 & Día 7 & Día 60 \\
\hline Leucocitos totales & 77,000 & 60,000 & 72,000 & 38,000 & 11,000 \\
\hline Eosinófilos & $4 \%$ & & $2 \%$ & $4 \%$ \\
\hline Basófilos & & & & & \\
\hline Baciliformes & $14 \%$ & $4 \%$ & $4 \%$ & $10 \%$ & $2 \%$ \\
\hline Segmentados & $24 \%$ & $67 \%$ & $58 \%$ & $35 \%$ & $42 \%$ \\
\hline Linfocitos & $12 \%$ & $10 \%$ & $10 \%$ & $26 \%$ & $36 \%$ \\
\hline Monocitos & $1 \%$ & $11 \%$ & $1 \%$ & $1 \%$ & $12 \%$ \\
\hline Blastos & $25 \%$ & $16 \%$ & $14 \%$ & $10 \%$ & $0 \%$ \\
\hline Mielocitos & $10 \%$ & $2 \%$ & $1 \%$ & $8 \%$ & $2 \%$ \\
\hline
\end{tabular}

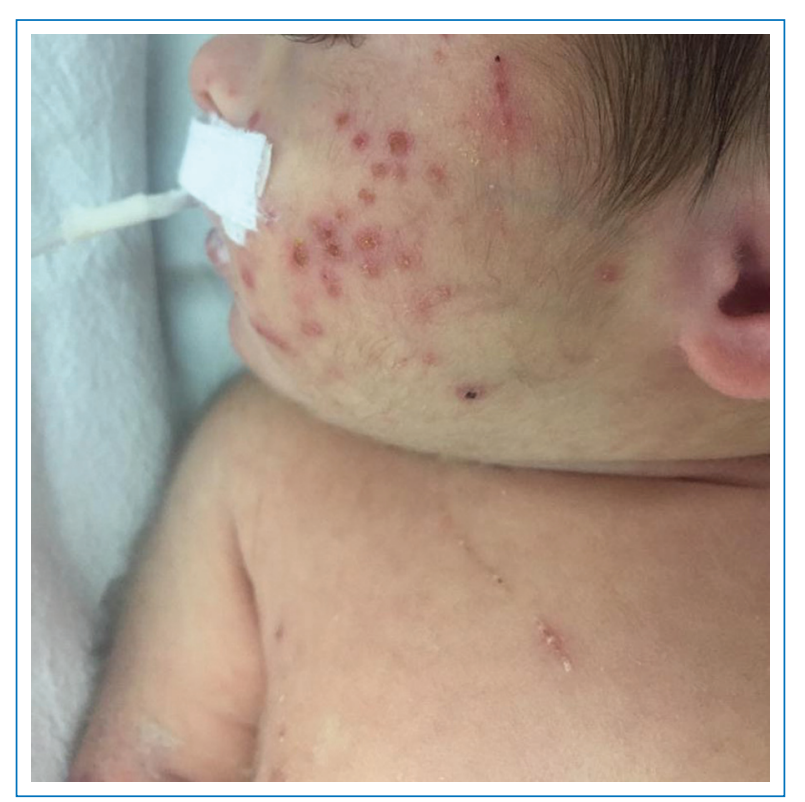

Figura 5. Caso 2. Lesiones vesículo-costrosas, algunas con costra de tipo melicérica, asentadas sobre una base eritematosa, ubicadas a nivel del rostro (mejillas).

La evolución también fue similar a la del paciente anterior, con normalización de los hemogramas y desaparición de las lesiones cutáneas alrededor del tercer mes de vida. En la actualidad, 18 meses después, continúa en seguimiento en el hospital con controles normales.

Resultados

Se incluyen en esta presentación dos casos característicos de niños con TMT y lesiones cutáneas, ambos con diagnóstico de síndrome de Down. En concordancia con lo descrito en las publicaciones médicas previas, ambos niños son de sexo masculino y desarrollaron la enfermedad en los primeros días de vida.

Las lesiones cutáneas se ubicaron en especial en tronco y rostro, con predominio de las formas papulares y pápulo-vesiculares, con algunas lesiones semejantes a las del impétigo. En términos sistémicos, ambos mostraron hiperleucocitosis y sólo el paciente del caso 1 desarrolló hepatomegalia. La citometría de flujo en ambos casos fue consistente con TMT y en la biopsia cutánea los dos recién nacidos tuvieron infiltración por blastos.

En el seguimiento de estos pacientes se advirtió a los tres meses de vida la resolución de las lesiones de la piel y los parámetros de laboratorio. Hasta ahora no han experimentado recaídas ni transformación a otras anomalías hematológicas.

\section{Discusión}

Los niños con SD tienen un riesgo 10 a 20 veces mayor de padecer trastornos hematológicos, sean formas malignas (leucemia) o trastornos benignos y autorresolutivos (reacciones leucemoides y TMT) ${ }^{2,4}$.

EI TMT es una forma autolimitada de la leucemia mielocítica aguda, caracterizada por aumento de blastos en médula ósea y sangre periférica, y hepatomegalia. Ocurre en alrededor de $4 \%$ a $10 \%$ de los pacientes nacidos con SD y, por su parte, el TMT es exclusivo de niños con esta anomalía de base (aunque es posible también en pacientes fenotípicamente normales con mosaicismo de trisomía 21) 2-4 $^{2-}$ 
En cuanto a su fisiopatología, en fecha reciente se han descrito mutaciones somáticas en el gen GATA1 (exón 2) en los pacientes con TMT y SD. Este gen, localizado en el cromosoma $X$, codifica a un factor de transcripción esencial para la hematopoyesis, y su mutación da lugar a una proteína alterada (GATA1 short) que actuaría al producir una desregulación de la megacariopoyesis. Sin embargo, este mecanismo por sí solo no explicaría el desarrollo de la enfermedad, sino que se trataría de la conjunción de la trisomía 21, la mutación en GATA1, más un factor genético aún desconocido ${ }^{2,5,6}$.

La presentación clínica del TMT es muy variable, pero la edad media de diagnóstico en general es de tres a siete días de vida. En alrededor del $10 \%$ de los casos no se presentan síntomas; el resto puede manifestarse con hepatomegalia, esplenomegalia, derrames serosos, fibrosis hepática, leucocitosis, trombocitopenia, entre otros ${ }^{2}$.

La afectación cutánea se ha descrito en pocos casos ( $5 \%$, aunque puede presentarse hasta en un $50 \%$ de los TMT), y se trata de manifestaciones inespecíficas, como erupciones eritematosas, costrosas o vesículo-pustulosas (en ocasiones del tipo similar a las del impétigo), que se asientan en particular sobre rostro, con extensión posterior a tronco y extremidades y menos a menudo a palmas, plantas y cuello. Hasta en un $25 \%$ de los casos puede aparecer en sitios expuestos a traumatismo ${ }^{4,7}$.

Estas lesiones suelen comenzar en los primeros días de vida (entre los días 1 y 22) o incluso ser congénitas, y desaparecer en general antes del tercer mes de vida. La biopsia cutánea puede mostrar infiltrados leucémicos perivasculares y dérmicos consistentes con leucemia cutánea. La afectación epidérmica en la leucemia cutánea es inusual, a diferencia del TMT en el que muchos casos muestran una exocitosis marcada de células mononucleares atípicas. Cuando hay vesículas, la infiltración leucémica se encuentra en la dermis subyacente. Es importante realizar un estudio citológico de vesículas tan pronto como sea posible para demostrar blastos positivos a la mieloperoxidasa, con el fin de establecer un diagnóstico temprano ${ }^{2,4}$.

Entre los diagnósticos diferenciales a considerar en estos pacientes figuran el eritema tóxico neonatal, acropustulosis infantil, melanosis pustular neonatal transitoria, miliaria pustulosa, histiocitosis de células de Langerhans, infecciones por Staphylococcus aureus o Listeria monocytogenes, herpes simple, varicela zóster, citomegalovirus, Candida spp., Malassezia spp., o escabiosis $^{2,5,7}$.
A pesar de que el TMT suele resolverse de modo espontáneo en algunos meses, los pacientes con recuentos elevados de blastos y afectación sistémica grave pueden requerir tratamiento quimioterapéutico, y el fármaco de elección es el arabinósido de citosina en dosis bajas. Además, a pesar de la naturaleza benigna y autorresolutiva del TMT, estos pacientes deben controlarse de por vida, ya que tienen mayor riesgo ( 20\%) de desarrollar leucemia mielogénica aguda, leucemia linfoblástica aguda o más a menudo leucemia megacariocítica aguda, en un tiempo de uno a cinco años ${ }^{2,6,7}$.

El interés de esta presentación radica en mostrar dos casos típicos de neonatos con una afección poco frecuente, pero que deben conocer el dermatólogo y el pediatra, a fin de evitar diagnósticos erróneos y tratamientos inadecuados o innecesarios, además de garantizar también un seguimiento periódico para detectar transformación maligna de manera temprana. Cabe destacar la importancia de la participación del médico dermatólogo, ya que la realización de un extendido de una lesión cutánea determina con rapidez el diagnóstico de una enfermedad sistémica como ésta.

\section{Financiamiento}

Los autores declaran no haber tenido financiamiento alguno para la realización de este trabajo.

\section{Conflicto de intereses}

Los autores declaran no tener conflicto de intereses.

\section{Responsabilidades éticas}

Protección de personas y animales. Los autores declaran que para esta investigación no se han realizado experimentos en seres humanos ni en animales.

Confidencialidad de los datos. Los autores declaran que han seguido los protocolos de su centro de trabajo sobre la publicación de datos de pacientes.

Derecho a la privacidad y consentimiento informado. Los autores han obtenido el consentimiento informado de los pacientes o sujetos referidos en el artículo. Este documento obra en poder del autor de correspondencia.

\section{Bibliografía}

\footnotetext{
1. Greco MF, Marín CC. Enfermedades neonatales. En: Larralde M, Abad E, Luna P, Boggio P, Ferrari B. Dermatología pediátrica, $3^{\circ}$ ed. Buenos Aires; 2021:35-36.

2. Narváez-Rosales V, Sáez de-Ocariz MS, Carrasco-Daza D, Ramírez-Dávila B, Orozco-Covarrubias L, Durán-McKinster C, et al. Neonatal vesiculopustular eruption associated with transient myeloproliferative disorder: report of four cases. Int J Dermatol. 2013 Oct;52(10):1202-9.
} 
Med Cutan Iber Lat Am. 2021;49(Suppl 1)

3. Nar I, Surmeli-Onay O, Aytac S, Talim B, Kiper PO, Boduroglu K, et al. Vesiculopustular eruption in neonatal transient myeloproliferative disorder. Indian J Pediatr. 2014 Apr;81(4):391-3.

4. Brazzelli V, Segal A, Bernacca C, Tchich A, Bolcato V, Croci G, et al Neonatal vesiculopustular eruption in Down syndrome and transient myeloproliferative disorder: a case report and review of the literature. Pediatr Dermatol. 2019 Sep;36(5):702-706.
5. Watanabe K. Recent advances in the understanding of transient abnormal myelopoiesis in Down syndrome. Pediatr Int. 2019 Mar;61(3):222-229.

6. Reyes ZS, Bashir W, Pathare A. Transient myeloproliferative disorder and Down syndrome: Is there a link? Sultan Qaboos Univ Med J. 2012 Nov;12(4):498-502.

7. Iwashita N, Sadahira C, Yuza Y, Yoshihashi H, Kondou M. Vesiculopustular eruption in neonate with trisomy 21 and transient myeloproliferative disorder. J Pediatr. 2013 Mar;162(3):643-4. 